\title{
Morphological changes of post-isolation of caprine pancreatic islet
}

\begin{abstract}
Pancreatic islet transplantation is commonly used to treat diabetes. Cell isolation and purification methods can affect the structure and function of the isolated islet cells. Thus, the development of cell isolation techniques that preserve the structure and function of pancreatic islet cells is essential for enabling successful transplantation procedures. The impact of purification procedures on cell function can be assessed by performing ultrastructure and in vivo studies. Thus, the aim of this study was to evaluate the effect of caprine islets purification procedure on islet cell ultrastructure and functional integrity prior to and postisolation/purification. The islets were isolated from caprine pancreas by using an optimized collagenase XI-S concentration, and the cells were subsequently purified using Euro-Ficoll density gradient. In vitro viability of islets was determined by fluorescein diacetate and propidium iodide staining. Static incubation was used to assess functionality and insulin production by islet cells in culture media when exposed to various levels of glucose. Pancreatic tissues were examined by using light microscopy, fluorescence microscopy, scanning, and transmission electron microscopy. In vivo viability and functionality of caprine islets were assessed by evaluating the transplanted islets in diabetic mice. Insulin assay of glucose-stimulated insulin secretion test showed that the insulin levels increased with increasing concentration of glucose. Thus, purified islets stimulated with high glucose concentration $(25 \mathrm{mM})$ secreted higher levels of insulin $(0.542$ 曲目.346 $\mathrm{gg} / \mathrm{L})$ than the insulin

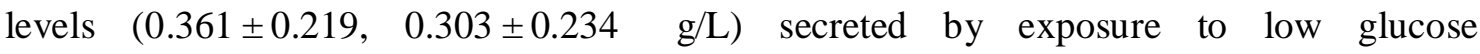
concentrations $(1.67 \mathrm{mM})$. Furthermore, insulin levels of recipient mice were significantly

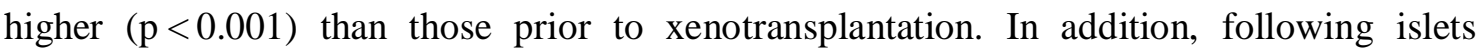
transplantation, there was significant enhancement in blood glucose levels of diabetic recipient mice. Overall, although the purified caprine islets had minor deformations in the plasma membrane and changes in cell integrity of peripheral region, the alterations did not significantly alter the functionality and viability of the purified islets.
\end{abstract}

Keyword: Caprine islet; Post-isolation; Purification; Ultrastructure; Islet functionality 